\title{
Optimization of Stir Casting Process Parameters for Producing MMC Aluminum Sacrificial Anode Incorporated with Manganese Dioxide Concentrate Powder
}

\author{
Abdelrahman Elsayed ${ }^{1}$, Adel Nofal ${ }^{2}$, Galal Attia ${ }^{3}$ \\ ${ }^{I}$ Teaching Assistant, Faculty of Petroleum and Mining Engineering, Suez University, Egypt. \\ ${ }^{2} \mathrm{PhD}$, Central Metallurgical Research and Development Institute-CMRDI, Egypt. \\ ${ }^{3}$ PhD, Faculty of Petroleum and Mining Engineering, Suez University, Egypt.
}

${ }^{3}$ ORCID: 0000-0001-6013-9803

\begin{abstract}
Owning to their markedly improved mechanical properties, aluminum metal matrix composites (AMMC's) have found a global approval as a reliable material for different applications. Nonetheless, researches on several reinforcements have proved that AMMC's properties also extend to show an improvement in electrochemical performance for being used as aluminum sacrificial anodes. Stir casting process is considered the most cost-efficient route for production of MMC's on industrial scale, the process itself is simple but requires fine tuning of the different processing parameters to obtain homogenous and high-quality cast products. In this research, stir casting process has been implemented and optimized for producing metal matrix composite of alloy $\mathrm{Al}-5 \% \mathrm{Zn}-0.1 \% \mathrm{Sn}$ incorporated with commercially available manganese dioxide concentrate for sake of manufacturing high performance aluminum sacrificial anodes under different solution treatment cycles. The electrochemical behavior of produced AMMC will be evaluated in separate publication as second part of the present work.
\end{abstract}

Keywords: stir casting, aluminum metal matrix composites (AMMC's), aluminum sacrificial anode, ceramic reinforcement.

\section{INTRODUCTION}

Incorporation of reinforcement particulates into aluminum and aluminum alloys, known as aluminum metal matrix composites (AMMC's), has dominated a wide variety of applications in aerospace, automotive and marine industries [1-2]. This could be related to the ability of AMMC's to overcome the drawback properties of aluminum alloys by offering a higher thermal and chemical stability, higher wear resistance and greater specific strength [3-4]. The reinforcements added to AMMC's to boost their mechanical properties are, and not limited to, $\mathrm{SiC}, \mathrm{B} 4 \mathrm{C}$, $\mathrm{BN}, \mathrm{TiC}, \mathrm{TiB} 2, \mathrm{ZrO} 2, \mathrm{Al} 2 \mathrm{O} 3$, graphite and carbon nanotubes [5-8]. However, advantages of AMMC's have not been restricted to enhancement of mechanical properties, Shibli et al. showed that incorporation of metal-oxides into Al-Zn alloys showed an improvement in its electrochemical properties when used as sacrificial anodes for cathodic protection purposes, several metal-oxides have been implemented for this target like $\mathrm{IrO} 2, \mathrm{CeO} 2, \mathrm{RuO} 2, \mathrm{ZnO}, \mathrm{Al} 2 \mathrm{O} 3$ and $\mathrm{MnO} 2$ [9-13].

Fabrication of metal matrix composites can be done by several techniques which can be categorized in three different routes, solid state route based on powder metallurgy technique, the liquid state route based on several casting techniques, and some other special manufacturing techniques which are infiltration methods [14-16]. Although solid state and infiltration techniques are considered more efficient in terms of final product soundness, property achievement and uniform microstructure distribution of reinforcements, liquid state techniques are yet more popular on the industrial scale due to process simplicity and ease of manufacturing besides being more economical solution for production of large components and mass scale production [17]. Amongst liquid state techniques, stir casting process is an extensively implemented method for production of MMC's by simply blending solid reinforcement mechanically into molten metal before allowing it to solidify in a suitable mold.

The straightforwardness and low cost of the process made it very popular for production of MMC's on industrial scale [18]. Nevertheless, this process simplicity is accompanied with some challenges mainly concerned with achieving fair degree of homogeneity in distribution of reinforcement particles and attaining good wetting between matrix and reinforcement particles, along with porosity defects in the final product due to air suction through the mechanically formed vortex [19].

The main factors which significantly affect stir casting process and determine the quality of the final product in terms of dispersion of reinforcement particles in molten metal and good interface bonding are shown in figure (1). For production of traditional or novel MMC's with high quality, all these factors need to be optimized together and processing conditions shall be fine-tuned to get the optimum results. 
International Journal of Engineering Research and Technology. ISSN 0974-3154, Volume 13, Number 10 (2020), pp. 2651-2659

(C) International Research Publication House. https://dx.doi.org/10.37624/IJERT/13.10.2020.2651-2659

Table 1 Examples of different processing parameters optimized for production of AMMC's from literature

\begin{tabular}{|c|c|c|c|c|c|c|}
\hline Matrix & Reinforcement & Reinforcement $\%$ & $\begin{array}{l}\text { Stirring speed } \\
(\mathrm{rpm})\end{array}$ & $\begin{array}{l}\text { Stirring time } \\
(\min )\end{array}$ & $\begin{array}{l}\text { Feeding } \\
\text { rate }(\mathrm{g} / \mathrm{s})\end{array}$ & Reference \\
\hline Al 7075 & $\mathrm{~B} 4 \mathrm{C}$ & 10 vol. $\%$ & 500 & NA & NA & [20] \\
\hline $\mathrm{Al} 6061$ & $\mathrm{~B} 4 \mathrm{C}$ & 10 wt. $\%$ & 500 & 5 & $0.9-1.5$ & [21] \\
\hline Al 7075 & graphite & 5 wt. $\%$ & 700 & 5 & $0.9-1.5$ & {$[21]$} \\
\hline Al 7075 & Graphite & $5,10,15,20$ wt. $\%$ & 500 & 5 & NA & {$[22]$} \\
\hline Al 6061 & Nano A12O3 & $0.5,1,1.5$ wt. $\%$ & 450 & 15 & NA & {$[23]$} \\
\hline A384 & $\mathrm{SiC}$ & 10 wt. $\%$ & $500,600,700$ & $5,10,15$ & NA & {$[24]$} \\
\hline A6061/1545K & $\mathrm{B} 4 \mathrm{C}$ & 5 wt. $\%$ & 450 & NA & NA & {$[25]$} \\
\hline LM25 & $\mathrm{B} 4 \mathrm{C}$ & $0,2,3$ wt. $\%$ & 550 & 10 & NA & {$[26]$} \\
\hline LM25 & $\mathrm{Al} 2 \mathrm{O} 3$ & $0,2,3$ wt. $\%$ & 550 & 10 & NA & [26] \\
\hline
\end{tabular}

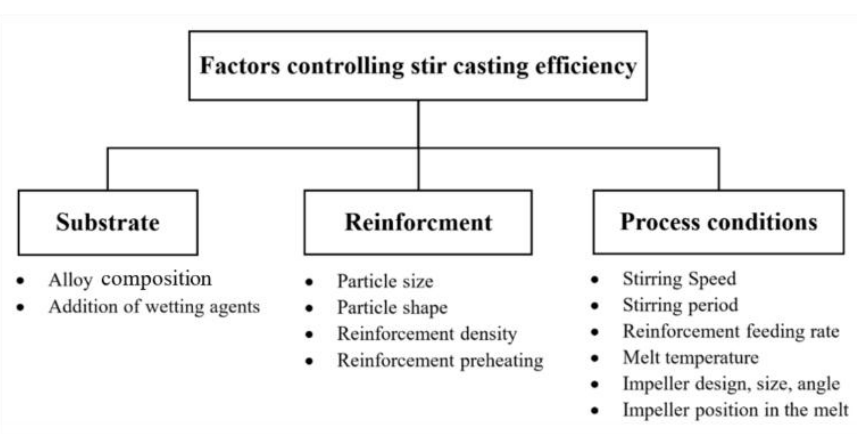

Figure 1. Factors affecting efficiency of stir casting process for high quality products

The optimized processing parameters for different matrices provided by some selected authors which are proved to give an efficient vortex for proper incorporation of different reinforcements are shown in table (1).

The aim of the present research is to study and optimize controlling parameters of stir casting process for production of AMMC with a substrate of $\mathrm{Al}-5 \% \mathrm{Zn}-0.1 \% \mathrm{Sn}$ reinforced with a powder of $\mathrm{MnO} 2$ concentrate for the purpose of producing high performance aluminum sacrificial anode using commercially available and low cost raw material. A master alloy of AMMC has been produced and then diluted to 4 different concentrations of reinforcement for electrochemical evaluation.

\section{MATERIALS AND METHODS}

\section{II.I Matrix Material Preparation}

Aluminum alloy $\mathrm{Al}-5 \% \mathrm{Zn}-0.1 \% \mathrm{Sn}$ has been processed from AA 1070 commercial pure aluminum $(0.182 \% \mathrm{Fe}, 0.037 \% \mathrm{Si}$, $0.047 \% \mathrm{Zn}$ ) obtained from the Aluminum Company of Egypt alloyed with $5 \%$ of special high grade zinc $(99.99 \%)$ and ultrapure tin $(99.995 \%)$. The purchased materials, which were in form of ingots, have been cut into small pieces using laboratory hacksaw before melting. Aluminum and alloying elements have been weighed using an electronic weighing balance.

\section{II.II Preparation of reinforcement material}

The commercially available manganese dioxide concentrate powder has been selected as the reinforcement material and has been supplied by Sinai Manganese Co. The manganese dioxide concentrate has been dried and ground using a rotating ball mill for 3 hours. Large particle size of the ground powder has been separated by using standard sieves until we reached size of around 5 micron which has been used as reinforcement. XRD analysis of manganese dioxide concentrate has been done to find out its constituents and their percentages. XRD diffraction pattern of manganese dioxide concentrate with percentage composition of its constituents are given in figure (2).

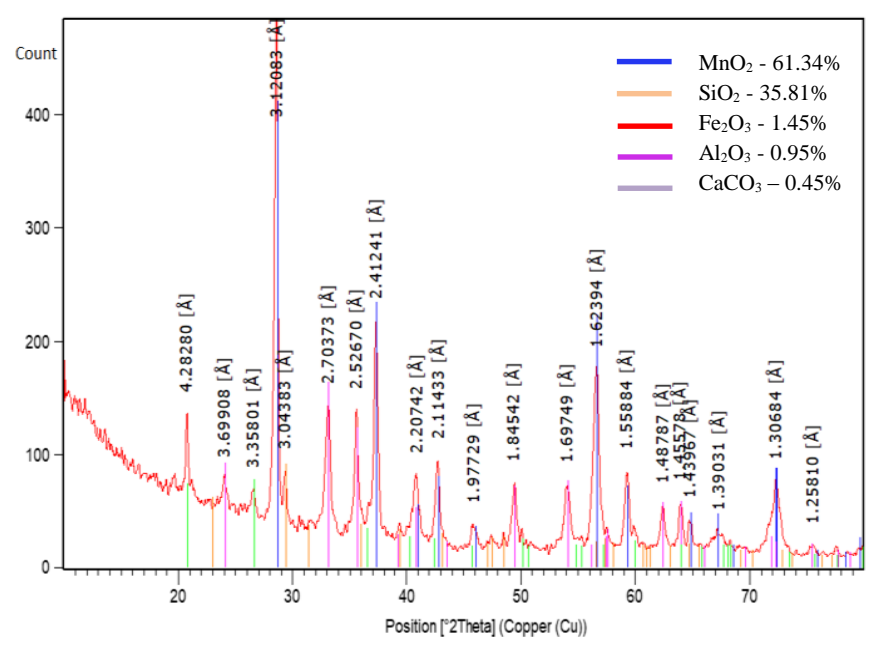

Figure 2. X-Ray diffraction pattern for metal-oxides mixture used as reinforcement for metal matrix composite

\section{II.III Stir casting setup}

Stir casting setup includes an electric resistance heating furnace that can reach up to a temperature of $1200^{\circ} \mathrm{C}$ comprising a top opening and cover as shown in figure (3). A graphite crucible is placed in the heating chamber with a feeder placed up at the center of the crucible for controlled addition of reinforcement powder. The setup is equipped with a motor with adjustable 
stirring speed of 300-1500 rpm and a stirring rode carried on a cantilever beam with controllable propeller height.

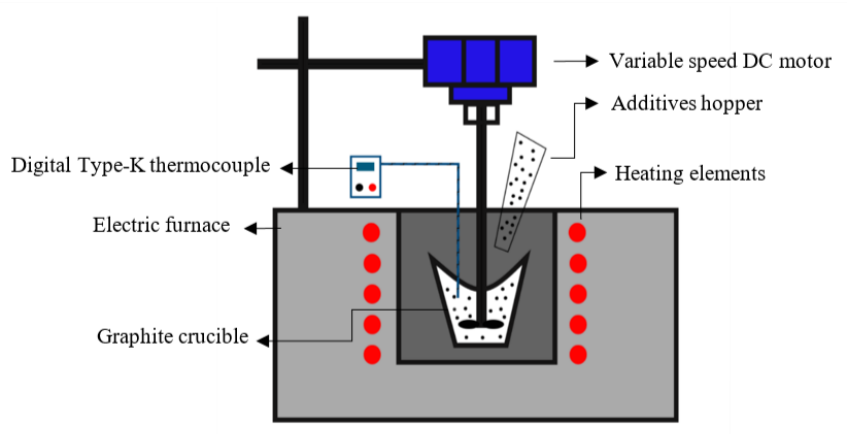

Figure 3. Schematic of stir casting setup

Propeller head is interchangeable to provide flexibility with using different stirring blades as shown in figure (3). A type-K thermocouple immersed in molten metal bath with accuracy of $\pm 1{ }^{\circ} \mathrm{C}$ was used for continuous temperature measurement of liquid alloy

\section{II.IV Stir casting process}

\section{II.IV.I Achieving wettability}

Reinforcement particles generally face two barriers to be incorporated into molten metal. The first is a mechanical barrier due to oxide layer on top of metal surface which can be reduced by skimming oxide layer prior to proceeding with particle addition. The second is a thermodynamic barrier which is described in terms of wettability, this barrier is quite more difficult to overcome as ceramic particles are by nature not wettable by liquid metals. The key solution for enhancing wettability is to reduce surface tension of the liquid melt to allow particles to unify with the matrix and avoid being repelled. Several turnarounds have been implemented to achieve this target, first of them is the addition of $\mathrm{Mg}$ in a percentage of $1 \%$ to the melt which was experimentally validated to improve wettability by acting as a surfactant reducing melt surface tension and by promoting solid-liquid interface reaction due to the high affinity of $\mathrm{Mg}$ for reaction with oxygen on surface of the incorporated particles [27-29]. However, the option of using $\mathrm{Mg}$ addition is not viable in our scope of this research as it would affect the electrochemical performance of final product which is to be used as sacrificial anodes.

Instead, another way of enhancing wettability is performing stirring process in the semi-solid state (mushy state) which increases melt viscosity and yields a lower surface tension and allows particles to be incorporated into the melt. Yet the melt in this case has very low fluidity and becomes not suitable for casting in final shapes, so it must be re-melted again into fully molten state to allow pouring in the final mold. Jasmi Hashim et al. have proved that performing stirring process in fully molten condition doesn't assist in particles incorporation and causes the reinforcement to float to the top of the melt [30].

\section{II.IV.II Stirring speed and time}

Stirring speed and stirring time are considered crucial parameters of stir casting process, and largely depend on size of particles and its amount added to the melt [24]. Stirring time less than 3 minutes doesn't give a chance for particles to be well distributed into the melt, longer stirring period is generally beneficial to improve incorporation efficiency. However, in our initial trials on different stirring times, we found a restriction on maximum stirring duration which originates from heat dissipation and drop in melt temperature. This decrease in melt temperature results in an intense rise in melt viscosity, which leads to foaming of the melt and higher losses in form of dross, accompanied with casting difficulty in into final mold. In this work, 5 minutes of stirring is adopted as an optimum stirring period and different stirring speeds of 300,700 and $1100 \mathrm{rpm}$ were applied to reach the optimum incorporation of the added powder.

\section{II.IV.III Stirrer design and dimensions}

Stirrer design is a key factor affecting the efficiency of stir casting process, the axial flow impellers are considered the best choice for solid suspension in liquids and blending liquids [31].

In a preliminary work, both propeller type stirrer and Pitchedblade turbine shown in figure (4) have been evaluated being the well-accepted impellers for solid suspension. Preliminary observation showed that propeller type stirrer produces a vigorous turbulent flow which results in splashing amount of liquid metal outside the crucible, meanwhile Pitched-blade turbine stirrer produces a smooth vortex facilitating particles incorporation. The convenience of Pitched-blade stirrer is referred to its unique flow pattern which is referred as a mixed flow, as it generates a flow in both axial and radial directions.

In the present work, a three Pitched-blade stirrer has been implemented. Blade angle has been proven to have a vital role in achieving homogenous distribution of reinforcement in the minimum stirring time, a simulation study by $\mathrm{S}$. Naher and et al. showed that optimum blade angle is 60 degrees to achieve the minimum stirring time for uniform incorporation [32]. Stirring blade is manufactured from mild steel sheet $2 \mathrm{~mm}$ thick, coated with a layer of vitreous enamel paint fired at $850^{\circ} \mathrm{C}$, enamel paint is considered a ceramic compound which excludes interaction between stirrer material and the molten metal.
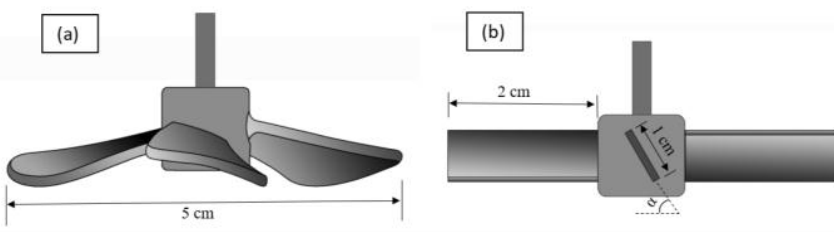

Figure 4. Design and dimensions of (a) propeller stirrer, (b) pitchedblade stirrer

\section{II.IV.IV Position of stirrer}

The position of stirring propeller has a significant contribution on the efficiency of the stirring process. As per Nagata observation, stirrer should not be located higher than $30 \%$ of melt height from bottom of the crucible to avoid accumulation of reinforcement particles at the bottom of the crucible [33]. This result is matching with the simulation results by Jasmi Hashim et al. showing that if the stirrer blade is placed at 0.1 and 0.7 of melt height, it will not give an efficient turbulence. If blade is positioned very close to the bottom of crucible there 
will be a dead zone with negligible fluid flow in the top segment of the melt and effect of stirring is observed only in the lower half of the crucible, while at the highest position dead zone is observed in the bottom half of the melt [34]. This is also matching with our preliminary trials which showed a vigorous turbulence of the melt if the propeller is placed at $70 \%$ and $50 \%$ from bottom of crucible and no formation of the required vortex.

\section{II.IV.V Feeding rate}

High feeding rate results in accumulation of reinforcement particles on top of the formed vortex and forms a barrier for particles to be withdrawn by the vortex into the melt making it difficult to achieve proper incorporation due formation of agglomerates of solid particles, which makes tuning of feeding rate crucial for optimal stirring performance. Researches have showed that feeding rate is preferred to be less than $48 \mathrm{~g} / \mathrm{min}$ and reaching a rate higher than $90 \mathrm{~g} / \mathrm{min}$ results in particles accumulation [34-35]. In this work feeding rate is set to be 9$10 \mathrm{~g} / \mathrm{min}$ fixed over the whole stirring period.

\section{II.IV.VI. Reinforcement preheating}

Preheating of reinforcement powder is necessary to eliminate moisture and any other existing gases or gases that could evolve during processing due to decomposition reactions of $\mathrm{CaCO}_{3}$ traces. This process is also considered vital as it can increase surface activity of particles and consequently increases its viability to be wetted by the melt and ease to be incorporated into the matrix [36]. In this work powder preheating is done in a muffle furnace at $400^{\circ} \mathrm{C}$ for 20 minutes just before addition to the melt.

Table (2) shows a summary of all process parameters implemented to incorporate manganese dioxide concentrate into $\mathrm{Al}-5 \% \mathrm{Zn}-0.1 \mathrm{Sn}$ melt.

Table 2: processing conditions for stir casting of MMC master alloy

\section{Processing Parameters}

\begin{tabular}{ll}
\hline Stirring Speed & $300,700,1100 \mathrm{rpm}$ \\
Stirring time & 5 minutes \\
Blade location inside the melt & At 0.3 of melt height \\
Reinforcement feeding rate & $9-10 \mathrm{~g} / \mathrm{min}$ \\
Stirring blade type & $\begin{array}{l}\text { Three pitched-blade axial } \\
\text { stirrer }\end{array}$ \\
Stirring blade angle & 60 degrees \\
Start melt temperature & $720^{\circ} \mathrm{C}$ \\
Finish melt temperature & $640^{\circ} \mathrm{C}$ \\
Re-melt temperature & $700^{\circ} \mathrm{C}$ \\
Re-melt time & $30 \mathrm{minutes}$ \\
\hline
\end{tabular}

\section{II.V Microstructure evaluation}

\section{II.V.I Optical microstructure analysis}

For evaluation of stir casting process and checking the effectiveness of incorporating metal-oxides and achieving the required wetting and homogeneity of distribution, microstructure samples have been taken from each of the investigated alloys and studied using optical microscope (Olympus). The samples for the metallographic examination were ground on SiC emery paper with grit number increasing from P240 to P2000 in sequence using water as a coolant. Fine polishing has been done for samples using 0.5 micron alumina powder on a rotating disc. No etching treatment was applied to avoid revealing intermetallic compounds for proper observation of the reinforcement particulates, Olympus stream motion software has been used to make image quantitative phase analysis to calculate volume fraction of oxides incorporated in the matrix in each case.

\section{II.V.II Scanning electron microscope analysis}

Samples from casting rods were metallographicaly prepared and examined using scanning electron microscope (Bruker), investigation has been done using secondary electron imaging, energy dispersive $\mathrm{x}$-ray analysis (EDX) and elemental mapping

\section{II.V.III Evaluation of reinforcement settling}

Microstructure samples have been taken from top and bottom of the vertically cast cylindrical rods to study the effect of gravitational settling force on particles concentration in the two ends of the rod. Gravitational settling is expected to take place due to the difference between densities of both incorporated particles and the parent matrix over the height of vertical rods or over melt height inside the crucible during stir casting remelt and during dilution process.

\section{RESULTS AND DISCUSSION}

\section{III.I Evaluation of stirring process efficiency}

After implementing processing parameter in table (3) at three different stirring speeds, results showed stirring at $700 \mathrm{rpm}$ gave the best incorporation, and the behavior of stirring at 300 rpm and $1100 \mathrm{rpm}$ was distinctively different as shown in figure (5). At stirring speed of $300 \mathrm{rpm}$, the vortex is not strong enough to withdraw and trap powder inside the melt which was apparent from vortex shape and its small depth which gave a low vortex momentum resulting in accumulation of reinforcement particles on top of the melt without being incorporated. Almost the contrary happened at stirring speed of $1100 \mathrm{rpm}$, the formed vortex was vigorous and its depth extended almost to the bottom of the crucible. This substantial vortex momentum resulted in not only withdrawal of particles, but led to producing a strong air suction that caused a significantly high oxidation rate driving a huge amount of liquid metal to be lost in form of dross.

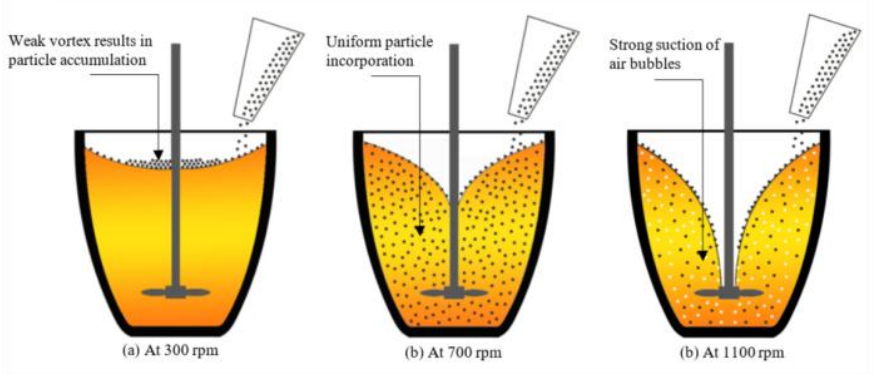

Figure 5 vortex shape and incorporation behavior at different stirring speeds, (a) at $300 \mathrm{rpm}$, (b) at $700 \mathrm{rpm}$ and (c) at $1100 \mathrm{rpm}$ 
The issue of dross is not limited to loss of molten metal (which was $40 \%$ of weight of the cast), but also extended to forming a barrier on top of the melt after a period of stirring which prevented particles from reaching the bulk of the metal and got trapped in the high viscosity dross layer. Figure (6-a) shows a picture of the resulted dross form stirring at $1100 \mathrm{rpm}$ and figure (6-b) shows the accumulated reinforcement particles on top of the melt due to stirring at $300 \mathrm{rpm}$.
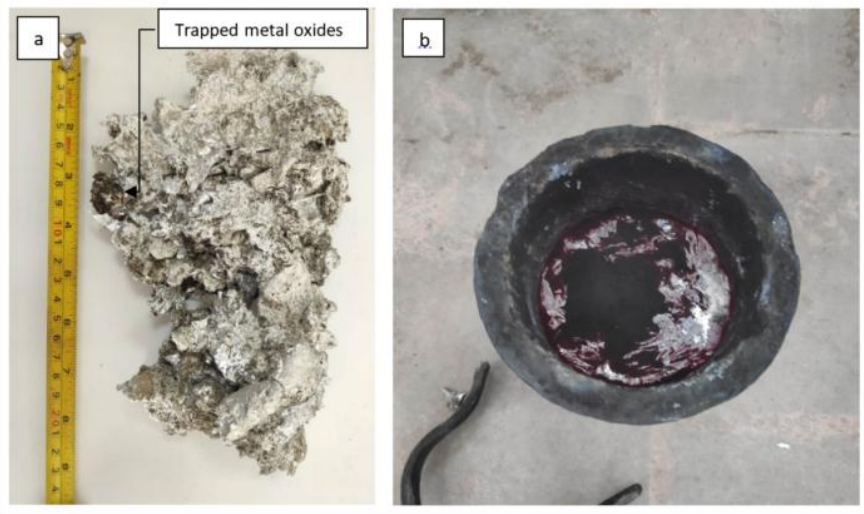

Figure 6. Resulting products of applying stirring speeds of $1100 \mathrm{rpm}$ and 300 $\mathrm{rpm}$, (a) formation of large amount of dross due to stirring at $1100 \mathrm{rpm}$ which shows trapping of oxides paricles. (b) accumulation of reinforcment on top of the melt after stirring at $300 \mathrm{rpm}$.

Quantitative metallography has been implemented for calculation of volume fraction of incorporated particle for 700 rpm casting condition using Olympus stream motion software. Microstructure images of final AMMC product shows an average of $10.2 \%$ volume fraction of homogeneously distributed particles in the matrix investigated at 5 different studied areas with a standard deviation of 2.55, figure (7) shows the distribution of incorporated particles
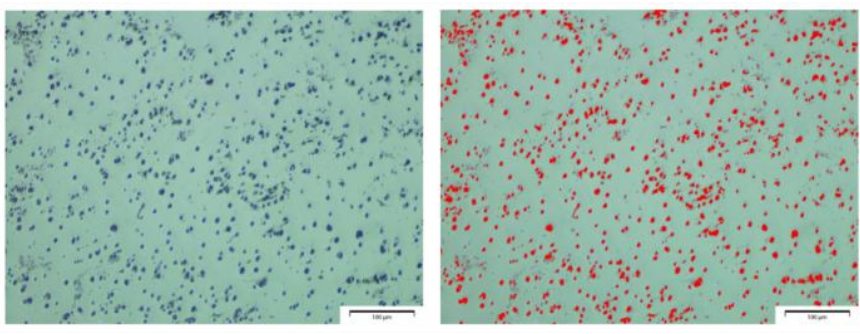

Figure 7 Evaluation of stir casting process efficiency in particle incorporation into the matrix (a) Microstructure image of master alloy after remelting shows distribution homogeneity of the incorporated particles at magnification of (b) Olympus stream motion quantitative phase analysis shows an average volume fraction of $10.2 \%$.

\section{III.II Microstructure evaluation of stir casting process}

After application of the process parameters mentioned in table (3) and at $700 \mathrm{rpm}$, microstructure samples show reasonable incorporation of metal-oxides particulates into the matrix as shown in figure (8). Microstructure samples taken from the cast before re-melting (which is not considered a final product yet) showed good incorporation and wetting of the incorporated particulates with some agglomerations of particles which can be explained by the dramatically high viscosity of the melt at the end of stirring process which hindered the flow of particles inside the melt. However, after re-melting at $700^{\circ} \mathrm{C}$ for 30 minutes without stirring samples showed a uniformly distributed particles inside the matrix which is mainly related to the decrease in melt viscosity providing freedom for particles to transport throughout the melt in addition to the effect of Brownian movement of liquid metal and its role in particle distribution and rearrangement [37]
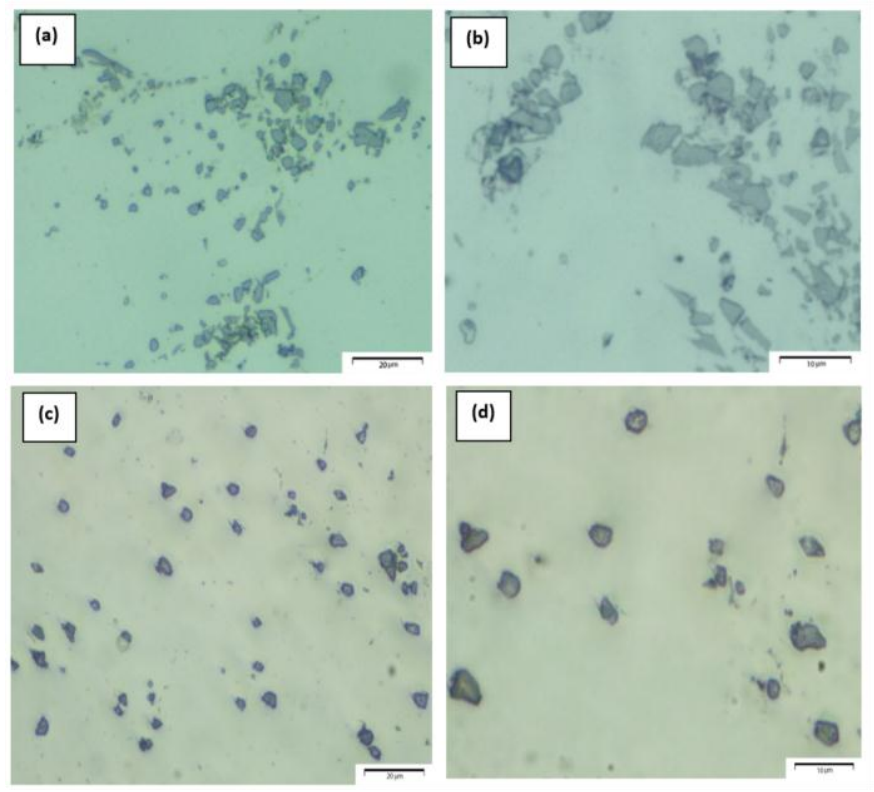

Figure 8: Microstructure images of master alloy (a \& b) show agglomeration of particles before re-melting at and 1000X respectively. (c \& d) show decent distribution of metal-oxides and fair degree of wetting with the matrix after re-melting at $500 \mathrm{X}$ and $1000 \mathrm{X}$ respectively.

\section{III.III Scanning electron microscope analysis}

Scanning electron microscope imaging could give more indepth understanding of behavior and interaction between incorporated metal-oxides and the matrix. EDX analysis of the incorporated particles showed no evidence for existence of $\mathrm{MnO} 2$ particles, X-ray point analysis of the incorporated particles showed it was mainly $\mathrm{Si}$ bearing oxide attributed to silica that existed in manganese dioxide concentrate powder as shown in figure (9). EDX elemental mapping has manifested this result by showing that $\mathrm{Mn}$ has been reduced and homogenously distributed in the matrix, and that particles' locations are mainly dominated by $\mathrm{Si}$ as shown in figure (10). According to M. A Taylor, manganese doesn't have any extent of solubility in aluminum matrix at room temperature; instead, a stable intermetallic compound Al6Mn with Orthorhombic structure is formed [38]. These findings can be interpreted in the light of interaction thermodynamics between molten aluminum matrix and incorporated oxides, thermodynamic stability of alumina is much higher than that of manganese dioxide which has led to an in-situ metallothermic reduction by aluminum during master alloy processing and master alloy dilution that gave free dissolved manganese in the matrix [3940]. This result is contradicting with the findings of Shibli et al. that showed $\mathrm{MnO} 2$ has been incorporated into the matrix without validating nature of inclusion using available characterization techniques [13]. However, the situation is way more different for silica which cannot be easily reduced by aluminum as it needs a temperature of around $1200{ }^{\circ} \mathrm{C}$ and holding time of 30-120 minutes to complete the reduction [41], these conditions of 
International Journal of Engineering Research and Technology. ISSN 0974-3154, Volume 13, Number 10 (2020), pp. 2651-2659

(C) International Research Publication House. https://dx.doi.org/10.37624/IJERT/13.10.2020.2651-2659
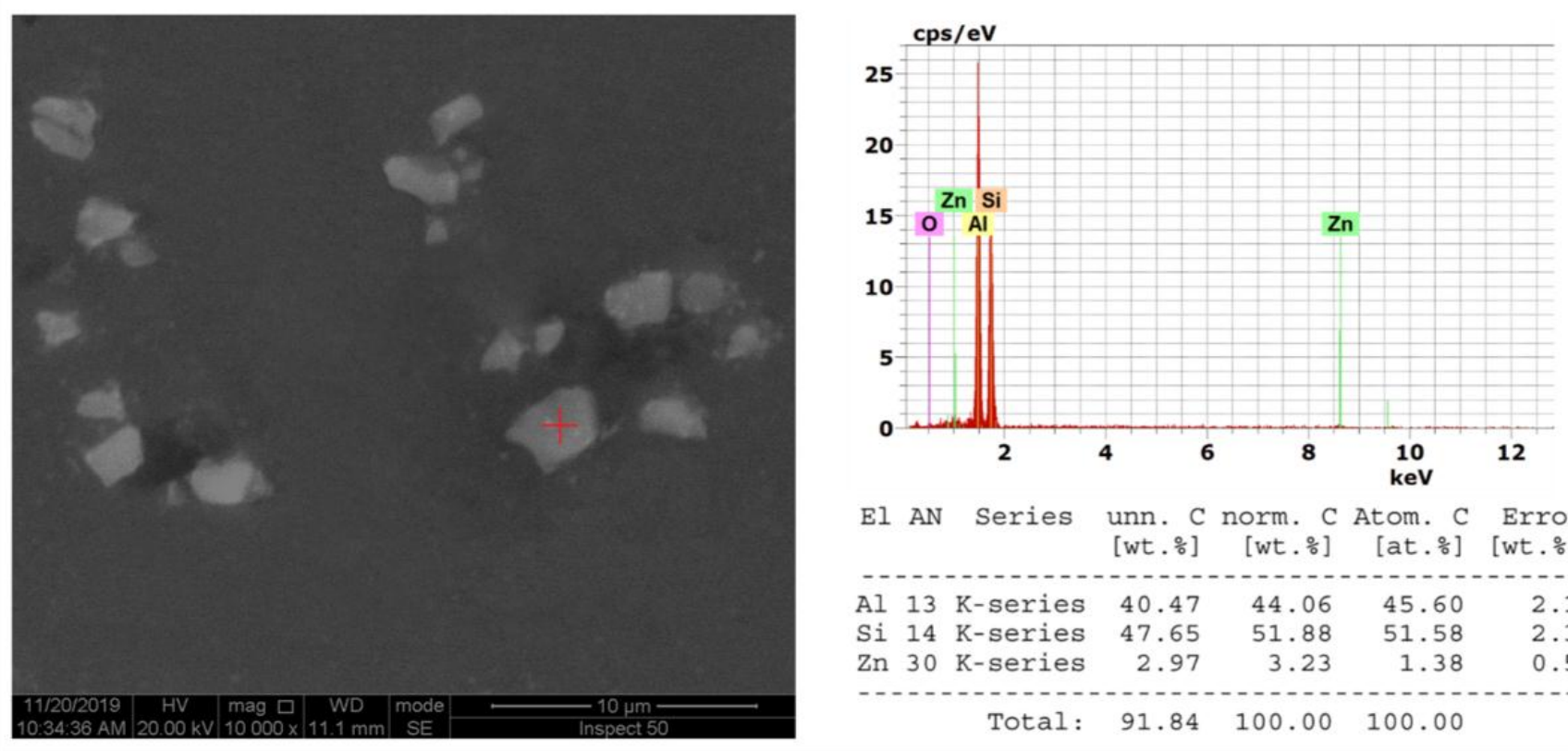

$\begin{array}{rrrrr}\text { El AN Series unn. C norm. C Atom. C } & \begin{array}{r}\text { Error } \\ \text { [wt. } \% \text { ] }\end{array} \text { [wt. } \% \text { ] } \\ \text { [at. } \% \text { [wt. } \% \text { ] }\end{array}$

Figure 9. Secondary electron image of incorporated particles with EDX point analysis
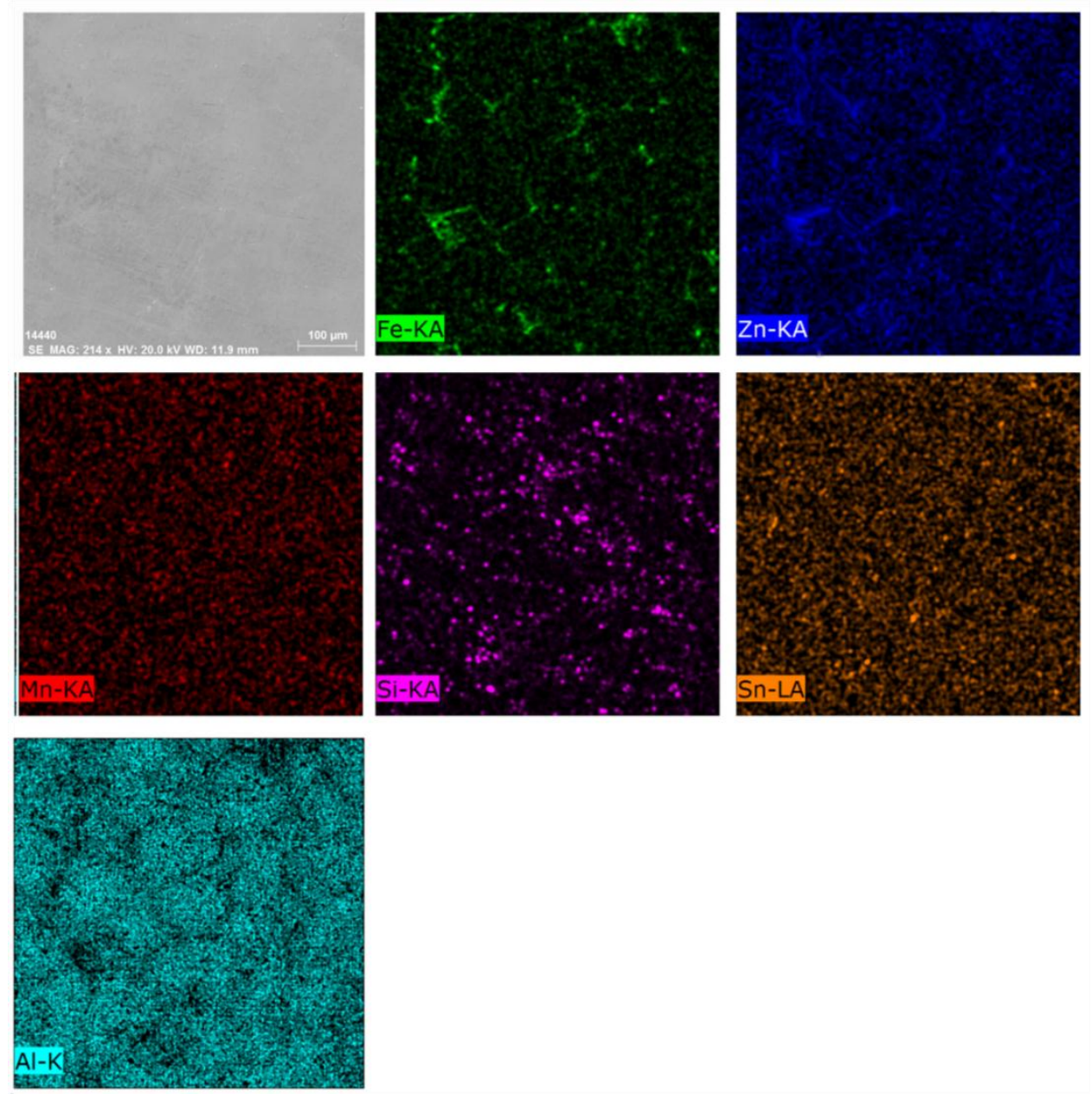

Figure 10. EDX elemental mapping after of final AMMC product after remelting 
superheating and mixing time are not provided during the adopted manufacturing process in this work. Secondary electrons image in figure (9) shows reasonable degree of wetting between silica particles and the matrix with no observation of any signs of decohesion or separation at the interface which proves efficiency of stir casting process regime followed in the present work.

\section{III.IV Evaluation of settling behavior}

The presence of silica particles in molten metal makes it susceptible to settling down under the gravitational force during master alloy re-melting or dilution process. Microstructure samples taken from top and bottom of the cast rods have showed fair degree of homogeneity of reinforcement particles as shown in figure (11). Olympus Stream Motion software was used to make quantitative phase analysis for both samples and showed that the volume fraction of particles in top and bottom samples is $8.6 \%$ and $8.2 \%$ respectively. This result can be interpreted by investigation of motion kinetics of particles present in the melt. There are three main forces acting on each particle, drag and buoyancy forces act on it in the upward direction while gravitational force acts on it downward [42]. Summing these three forces using Stock's and Newton's laws, the settling velocity of each particulate can be expressed in the following equation:

$V_{p}=\frac{\left(\rho_{\mathrm{p}}-\rho_{m}\right) d^{2} \mathrm{~g}}{18 \mu}$

Where $\mathrm{Vp}$ is the settling velocity in $\mathrm{m} / \mathrm{s}$ of each particulate, $\mathrm{d}$ is diameter of the particle in $\mathrm{m}, \mu$ is viscosity of molten metal in Pa.s, $\rho_{\mathrm{p}}$ and $\rho_{m}$ are the densities of particles and molten metal respectively in $\mathrm{Kg} / \mathrm{m}^{3}$, and $\mathrm{g}$ is the gravitational acceleration $\left(9.81 \mathrm{~m} / \mathrm{s}^{2}\right)$. By substituting the value of $\rho_{\mathrm{p}}$ and $\rho_{m}$ as $2.65 \mathrm{~g} / \mathrm{cm} 3$ and $2.375 \mathrm{~g} / \mathrm{cm} 3$ respectively, the viscosity of molten aluminum at $700{ }^{\circ} \mathrm{C}$ is $1.235 \mathrm{mPa} . \mathrm{S}$ [43], and for average particle diameter of 5 microns, we get a settling velocity of $180 \mu \mathrm{m} / \mathrm{min}$. This very low settling rate means that over the whole re-melting or dilution process which lasted for 30 minutes each, particles have undergone settling for a distance of only $5.4 \mathrm{~mm}$ inside the crucible which is expected to be rectified by the turbulence resulted during pouring of molten metal into the vertical mold. In addition to density and size, settling rate also depends on volume fraction of particles introduced to the melt which created hindrance to settling kinetics due to interaction with surrounded particles. The hindered settling has been modelled by Richardson and Zaki who have shown that hindered settling velocity is reduced to be $V_{c}=V_{0}(1-f)^{n}$ where, Vo is the Stokes's settling velocity, $\mathrm{f}$ the volume fraction of reinforcement particles, and $\mathrm{n}$ is a factor that depends on the Reynolds number, particle diameter and the container diameter [44].

\section{III.V Optimization of process parameters}

Figure (12) represents the optimum production sequence used in the present study. The optimum processing conditions for obtaining homogeneous distribution of the reinforcement particles during synthesis of the investigated metal matrix composite by stir casting without addition of any wetting agents are as following:

- Feeding rate $9-10 \mathrm{~g} / \mathrm{min}$.

- $\quad$ Stirring speed at $700 \mathrm{rpm}$

- Stirring period of 5 minutes.

- Three pitched-blade stirring impeller is recommended.

- $\quad$ Stirring blade angle of 60 degrees.

- Start melt temperature before stirring of $720^{\circ} \mathrm{C}$.

- Finish melt temperature after of stirring of $640^{\circ} \mathrm{C}$.

- Metal matrix composite shall be re-melted at $700^{\circ} \mathrm{C}$.

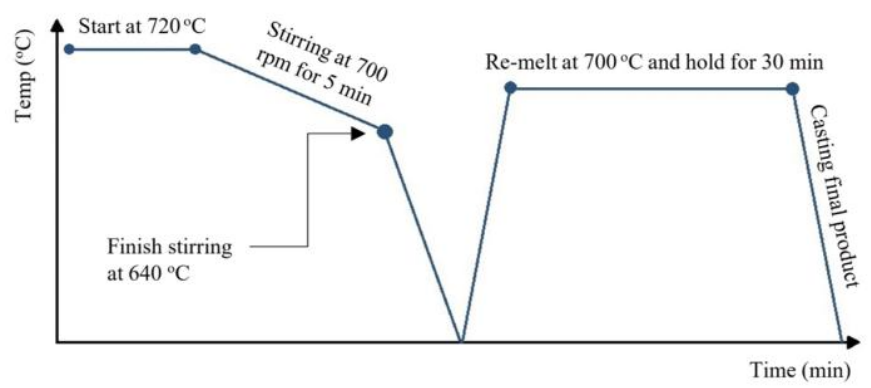

Figure 12. represent the optimum production sequence used in the present study
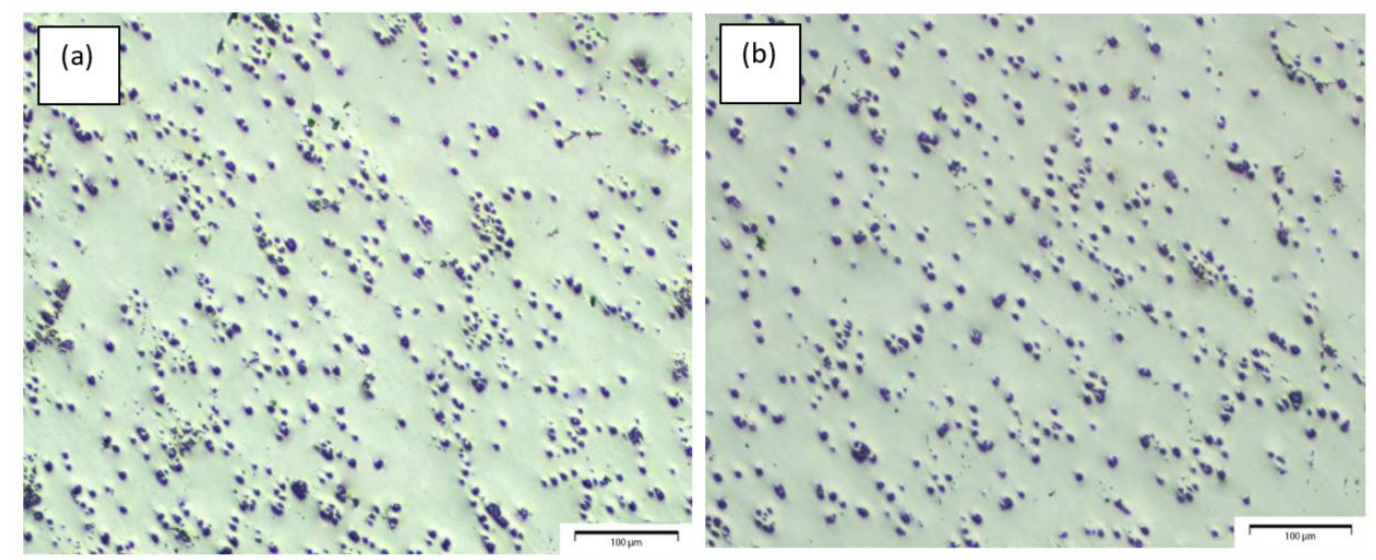

Figure 11: Microstructure images showing uniformity of particles distribution over the vertically cast rods at magnification of 100X (a) Top sample, (b) bottom sample 


\section{CONCLUSION}

On the basis of the investigation of Al-5Zn-0.1Sn MMC incorporated with manganese dioxide concentrate, the following conclusions can be drawn:

1. The optimal stir casting parameters has been determined as: stirring speed of $700 \mathrm{rpm}$, stirring time of $5 \mathrm{~min}$, federate of $10 \mathrm{~g} / \mathrm{min}$. and three pitched-blade stirring impeller is recommended.

2. Re-melting MMC is considered a crucial step to achieve the desired homogeneity of distribution assisted by Brownian motion of the melt which contributes to particles rearrangement.

3. EDX elemental mapping analysis proved that manganese dioxide particles are reduced by molten aluminum and dissolved in the alloy by a metallothermic reaction.

4. Settling phenomenon of $\mathrm{SiO} 2$ particles is very slow and can be neglected being not considerably affecting distribution of reinforcement particles studied at top and bottom locations of the vertically cast cylindrical rods.

\section{ACKNOWLEDGMENT}

Authors are grateful to the staff of the pilot casting unit Central Metallurgical Research and Development Institute - CMRDI for dedicating their time and effort to facilitate this research work.

\section{REFERENCES}

[1] F. J. L. Alves, A. M. Baptista, and A. T. Marques, 2016. Metal and ceramic matrix composites in aerospace engineering. Advanced Composite Materials for Aerospace Engineering .Elsevier Ltd, 59-99.

[2] K. K. Chawla and N. Chawla, 2014. Metal Matrix Composites : Automotive Applications, Encycl. Automot. Eng., pp. 1-6.

[3] D. K. Das, P. C. Mishra, S. Singh, and R. K. Thakur, Properties of ceramic-reinforced aluminium matrix composites - a review, Int. J. of Mechanical Mater. Eng., vol. 1:12, pp. 1-16, 2014.

[4] A. M. . Hamouda and M. S. . Hashmi, 1996. Mechanical properties of aluminum metal matrix composites under impact loading, Mater. Technol. Process., vol. 36, no. 95.

[5] A. Baradeswaran, S. C. Vettivel, A. E. Perumal, N. Selvakumar, and R. F. Issac, 2014. Experimental investigation on mechanical behaviour, modelling and optimization of wear parameters of B4C and graphite reinforced aluminium hybrid composites, J. Mater., vol. 63, pp. 620-632.

[6] S. Suresha and B. K. Sridhara, 2010. Wear characteristics of hybrid aluminium matrix composites reinforced with graphite and silicon carbide particulates, Compos. Sci. Technol., vol. 70, no. 11, pp. 1652-1659.
[7] M. K. Sahu, A. Valarmathi, S. Baskaran, and V. Anandakrishnan, 2014. Multi-objective optimization of upsetting parameters of $\mathrm{Al}-\mathrm{TiC}$ metal matrix composites : A grey Taguchi approach, J. Eng. Manuf., vol. 228, no. 11, pp. 1501-1507.

[8] R. Keshavamurthy, S. Mageri, G. Raj, B. Naveenkumar, P. M. Kadakol, and K. Vasu, 2013. Microstructure and Mechanical Properties of Al7075-TiB 2 in-situ composite, Res. J. Mater. Sci., vol. 1, no. 10, pp. 6-10.

[9] S. M. A. Shibli and V. S. Gireesh, 2003. Surface activation of aluminium alloy sacrificial anodes by IrO 2, Appl. Surf. Sci., vol. 219, no. 3-4, pp. 203-210.

[10] S. M. A. Shibli, S. R. Archana, and P. Muhamed Ashraf, 2008. Development of nano cerium oxide incorporated aluminium alloy sacrificial anode for marine applications, Corros. Sci., vol. 50, no. 8, pp. 2232-2238.

[11] S. M. A. Shibli and S. George, 2007. Electrochemical impedance spectroscopic analysis of activation of Al- $\mathrm{Zn}$ alloy sacrificial anode by RuO 2 catalytic coating, Appl. Surf. Sci., vol. 253, no. 18, pp. 7510-7515.

[12] S. M. A. Shibli, B. Jabeera, and R. Manu, 2007. Development of high performance aluminium alloy sacrificial anodes reinforced with metal oxides, Mater. Lett, vol. 61, no. 14-15, pp. 3000-3004.

[13] S. M. A. Shibli and K. K. Binoj, 2009. Development of $\mathrm{MnO} 2$-incorporated high performance aluminum alloy matrix sacrificial anodes, J. Appl. Electrochem., vol. 39, no. 2, pp. 159-166.

[14] D. K. Koli, G. Agnihotri, and R. Purohit, 2013. Properties and Characterization of Al-Al2O3 Composites Processed by Casting and Powder Metallurgy Routes ( Review ), Int. J. Latest Trends Eng. Technol., vol. 2, no. 4, pp. 486-496.

[15] K. M. S. Manu, L. A. Raag, T. P. D. Rajan, M. Gupta, and B. C. Pai, 2016. Liquid Metal Infiltration Processing of Metallic Composites : A Critical Review, Metall. Mater. Trans. B, no. Mmc.

[16] M. T. Sijo and K. R. Jayadevan, 2016. Analysis of stir cast aluminium silicon carbide metal matrix composite : A comprehensive review, Procedia Technol., vol. 24, pp. 379-385.

[17] W. C. Harrigan, 1998. Commercial processing of metal matrix composites, Mater. Sci. Eng. A, vol. 244, no. 1, pp. 75-79.

[18] S. Suresh and N. S. V. Moorthi, 2013. Process development in stir casting and investigation on microstructures and wear behavior of TiB2 on A16061 MMC, Procedia Eng., vol. 64, pp. 1183-1190.

[19] U. K. Annigeri and G. B. Veeresh, 2017. Method of stir casting of Aluminum metal matrix Composites : A review, Mater. Today Proc., vol. 4, no. 2, pp. 1140-1146.

[20] A. Baradeswaran and A. E. Perumal, 2013. Influence of $\mathrm{B} 4 \mathrm{C}$ on the tribological and mechanical properties of Al 7075-B4C composites, Compos. PART B, vol. 54, pp. 146-152.

[21] F. I. R. Baradeswaran A, Vettivel SC, Elaya Perumal A, Selvakumar N, 2014. Experimental investigation on 
mechanical behaviour, modelling and optimization of wear parameters of $\mathrm{B} 4 \mathrm{C}$ and graphite reinforced aluminium hybrid composites, Mater. Des., no. 63, pp. 620-632.

[22] [A. Baradeswaran and A. E. Perumal, 2014. Wear and mechanical characteristics of Al 7075/graphite composites, Compos. Part B Eng., vol. 56, no. August, pp. 472-476.

[23] H. Reza, S. Abolkarim, M. Haddad, and Y. Huang, 2014. Investigation of microstructure and mechanical properties of Al6061-nanocomposite fabricated by stir casting, J. Mater., vol. 55, pp. 921-928.

[24] S. B. Prabu, L. Karunamoorthy, S. Kathiresan, and B. Mohan, 2006. Influence of stirring speed and stirring time on distribution of particles in cast metal matrix composite, Journal of Materials Processing Technology.vol. 171, pp. 268-273,

[25] B. Vijaya Ramnath, C. Elanchezhian, M. Jaivignesh, S. Rajesh, C. Parswajinan, and A. Siddique Ahmed Ghias, 2014. Evaluation of mechanical properties of aluminium alloy-alumina- boron carbide metal matrix composites, Mater. Des., vol. 58, pp. 332-338.

[26] A. V Pozdniakov, V. S. Zolotorevskiy, R. Y. Barkov, A. Lotfy, and A. I. Bazlov, 2016. Microstructure and Material Characterization of 6063/B4C and 1545K/B4C Composites Produced by Two Stir Casting Techniques for Nuclear Applications A.V., J. Alloys Compd., no. 664, pp. 317-320.

[27] R. M. Pillai, 1995. Review Role of magnesium in cast aluminiurn alloy matrix composites, J. Mater. Sci., vol. 30, pp. 1903-1911.

[28] O. F. Materials, 1993. The wettability and the reaction for SiC particle/AI alloy system, J. Mater. Sci., vol. 28, pp. 684-690.

[29] L. Poovazhagan, K. Rajkumar, P. Saravanamuthukumar, S. Javed Syed Ibrahim, and S. Santhosh, 2015. Effect of Magnesium Addition on Processing the Al-0.8 Mg-0.7 $\mathrm{Si} / \mathrm{SiC}_{\mathrm{p}}$ Metal Matrix Composites, Appl. Mech. Mater., vol. 787, pp. 553-557.

[30] J. Hashim, L. Looney, and M. S. J. Hashmi, 2001. The enhancement of wettability of $\mathrm{SiC}$ particles in cast aluminium matrix composites, vol. 119, pp. 329-335.

[31] E. L. Paul, V. A. Atiemo-obeng, and S. M. Kresta, 2004. Handbook of industrial mixing. New Jersey: John Wiley \& Sons,

[32] S. Naher, D. Brabazon, and L. Looney, 2003. Simulation of the stir casting process, vol. 144, pp. 567-571.

[33] S. Nagata, 1976. Mixing-principles and applications. New York: Wiley.

[34] J. Hashim, L. Looney, and M. S. J. Hashmi, 2002. Particle distribution in cast metal matrix composites Part II, vol. 123, no. January, pp. 258-263.

[35] V. Auradi, G. L. Rajesh, and S. A. Kori, 2014. Processing of B4C Particulate Reinforced 6061Aluminum Matrix Composites by melt stirring involving two-step addition, Procedia Mater. Sci., vol. 6, no. Icmpc, pp. 1068-1076.
[36] S. Suresha and B. K. Sridhara, 2010. Effect of silicon carbide particulates on wear resistance of graphitic aluminium matrix composites, Mater. Des, vol. 31, no. 9, pp. 4470-4477.

[37] W. B. Russel, 1981. Brownian motion of small particles suspended, Annu. Rev. Fluid Mech., vol. 13, pp. 425455.

[38] M. A. Taylor, 1960. Intermetallic phases in the aluminium-manganese binary system, Acta Metall., vol. 8, no. 4, pp. 256-262.

[39] S. Jiaxing, G. Tao, D. Wen, M. Yiming, F. Xiang, and W. Hao, 2018. Study on Thermal Chemical Reaction of Al / MnO 2 Thermite, Earth Environ. Sci., vol. 186.

[40] B. SARANG, A. SARANG, and H. S. RAY, 1996. Kinetics of Aluminothermic moanalytical Investigation Reduction of Mn02 and Fe203: A Thermoanalytical Investigation, ISIJ Int., vol. 36, no. 9, pp. 1135-1141.

[41] Dosajet al., 2014. Methods and system for producing aluminum-silicon alloy, US Pat. No. 8900341 B2.

[42] A. S. Negi and T. Shanmugasundaram, 2020. Hybrid particles dispersion strengthened aluminum metal matrix composite processed by stir casting, Mater. Today Proc.

[43] M. J. Assael et al., 2006. Reference Data for the Density and Viscosity of Liquid Aluminum and Liquid Iron, $J$. Phys. Chem. Ref. Data, vol. 35, no. 1.

[44] J. Richardson and W. Zaki, 1954. SEDIMENTATION AND FLUIDISATION : PART I, TRANS. INSTN CHEM. ENGRS, vol. 32, pp. 35-53. 